\title{
CMOS X-rays detector array based on scintillating light guides
}

\author{
J.G. Rocha ${ }^{\text {a,* }}$, N.F. Ramos ${ }^{\text {a }}$, S. Lanceros-Mendez ${ }^{\text {b }}$, R.F. Wolffenbuttel ${ }^{\text {c }}$, J.H. Correia ${ }^{\text {a }}$ \\ a Department of Industrial Electronics, University of Minho, Campus de Azurem, 4800-058 Guimaraes, Portugal \\ ${ }^{\mathrm{b}}$ Department of Physics, University of Minho, Campus de Gualtar, 4710-057 Braga, Portugal \\ ${ }^{\mathrm{c}}$ Laboratory for Electronic Instrumentation, Delft University of Technology, Mekelweg 4, 2628 CD Delft, The Netherlands
}

Received 20 September 2002; received in revised form 5 September 2003; accepted 24 September 2003

\begin{abstract}
This paper describes a pixel imaging array consisting in $400 \mu \mathrm{m} \times 400 \mu \mathrm{m}$ photodiodes fabricated in CMOS technology. An array of scintillating CsI:Tl crystals is placed above the photodiodes. These crystals are encapsulated in aluminum walls, forming a light path that guides the visible light produced by the scintillating crystal into the photodiodes. In this way, the X-ray energy is first converted into visible light which is then detected by the photodiode at the end of each light guide. The scintillator is $800 \mu \mathrm{m}$ thick, absorbing almost all $20 \mathrm{keV}$ $\mathrm{X}$-ray photons. Usually, the spatial resolution of the scintillating X-ray detectors is identical to the scintillator thickness. By using the light guides, the scintillator thickness can be increased without decreasing the spatial resolution. The increase of the scintillator thickness is desirable in order to increase the X-rays absorption efficiency. Tests carried out on the system show very promising results near 20 keV. (c) 2003 Elsevier B.V. All rights reserved.
\end{abstract}

Keywords: X-rays; Scintillator; Digital radiography

\section{Introduction}

The digital X-ray imaging systems are now replacing silver films (traditional radiography) in a large number of fields, enabling real time image acquisition and processing and eliminating the costs and the pollution caused by the silver films. One of the first digital X-ray imagers was based on a silicon charge coupled device (CCD). The silicon has a low $\mathrm{X}$-ray absorption coefficient, but for each $1 \mathrm{keV}$ of X-ray photons absorbed, about 277 electrons are excited. Therefore images with sufficient quality for diagnostics with a radiation dose slightly smaller than the required for silver films can be achieved. However, the small number of photons detected by the CCD results in a significant quantum noise. There are two methods to reduce the quantum noise in the sensors: either the radiation dose can be increased or the quantum efficiency of the sensor can be improved. The increase of the X-ray dose is obviously not desired for medical applications.

The quantum efficiency of the sensor can be increased by adding a scintillating layer above the CCD. Since the $\mathrm{X}$-rays are first absorbed by the scintillating layer, which has a high absorption coefficient, and then converted into

\footnotetext{
* Corresponding author. Tel.: +351-253510190; fax: +351-253510189. E-mail address: gerardo@dei.uminho.pt (J.G. Rocha).
}

visible (or near visible) light, the quantum efficiency of the detector is increased. The problem of this method is that the spatial resolution of the device is approximately equal to the thickness of the scintillating layer (Fig. 1(a)).

In order to increase the thickness of the scintillating layer without decrease spatial resolution, scintillating light guides separated by reflective walls can be used, as is shown in Fig. 1(b) [1]. In this case, the light yield by each scintillator is guided to the corresponding CCD area by the reflective layers. The spatial resolution becomes approximately equal to the distance between the reflective layers. A similar approach uses the CsI:Tl scintillator grown with a columnar structure which is formed when evaporated under special conditions [2,3]. This structure also guides the light yield into the CCD (or another photodetector), but it has some limitations, such as:

- The CsI:Tl must be evaporated and annealed. This fabrication step may not be compatible with the photodetector technology.

- Other scintillating materials, interesting for some applications, do not form a columnar structure when evaporated, therefore they cannot be used.

Our approach uses CMOS technology instead of a CCD. The recent development in CMOS image detectors opens a new way to build digital X-rays imagers. The replacement of CCDs with CMOS detectors is desirable for several reasons: 


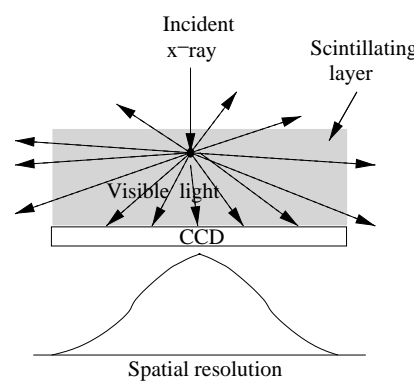

(a)

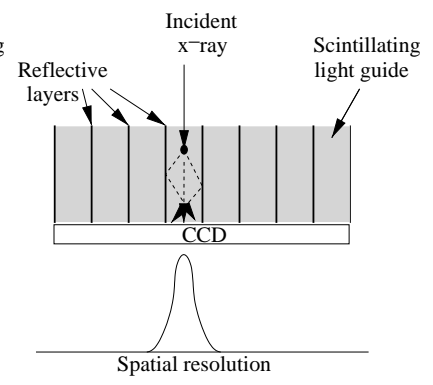

(b)
Fig. 1. Effect of reflective layers on the spatial resolution: (a) a single scintillating layer; (b) scintillating light guides.

- The operating power of CMOS is 5-10 times lower than the operating power for CCD with processing electronics.

- The CMOS is a standard fabrication process, while CCD requires special manufacturing.

- CMOS fabrication costs are 5-10 times lower.

- It is possible to integrate analog and digital processing electronics in CMOS.

The achievement of both low power and low cost is highly desirable for portable applications as well as situations where large X-ray imaging machines are required. The drawback is that it is difficult to match the high performance characteristics of CCD in terms of image quality [4].

\section{System design}

In this work, a new type of detector based on scintillating guides with reflective layers and CMOS readout has been developed. The first prototype consists on a $2 \times 2$ array of photodiodes with a scintillating light guide above each one. Fig. 2 shows a schematic diagram of the detector. The scintillating light guides consist on scintillating crystals embedded into aluminum cavities. The scintillating crystal converts the X-rays into visible light, which is then converted to an electrical signal by the CMOS photodiode [5]. Each scintillator is isolated from its neighbors by the high-reflective aluminum walls, which allow multiple reflections (Fig. 1) and guides the produced visible light through the scintillator (transparent for the visible light) to the photodiodes. More-

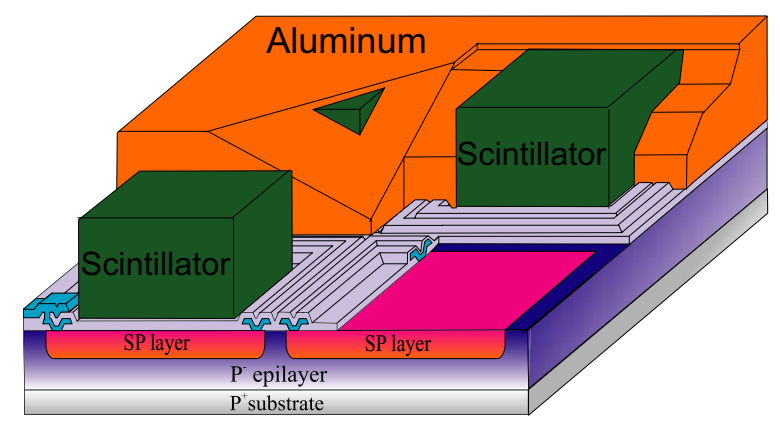

Fig. 2. Sensor structure for an array of $2 \times 2$ pixels.

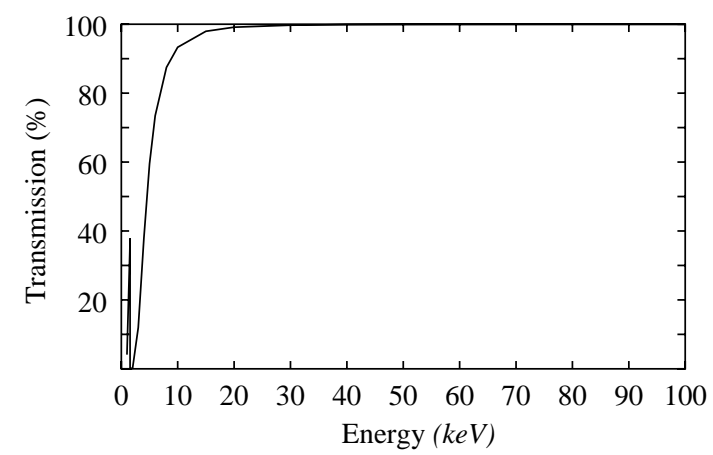

Fig. 3. Transmission of a $10 \mu \mathrm{m}$ thick aluminum film from 1 to $100 \mathrm{keV}$.

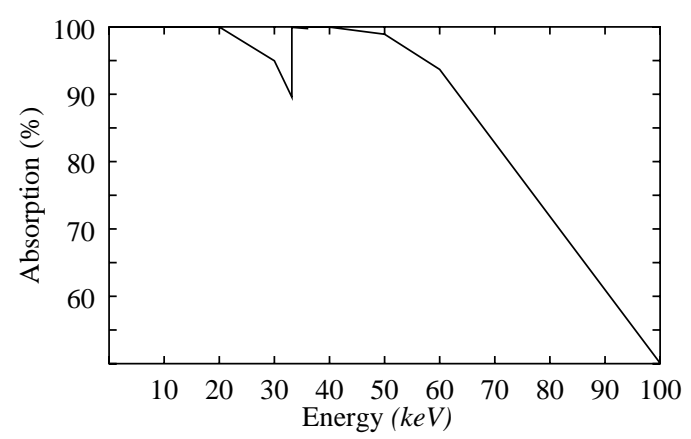

Fig. 4. Absorption of a $800 \mu \mathrm{m}$ thick CsI scintillating crystal from 1 to $100 \mathrm{keV}$.

over, introducing a reflective layer above the scintillator (in the X-rays path) confines the light inside the light guide, increasing the efficiency. The X-ray photons interact weakly with the reflective layer placed above the scintillating crystals, since it is constituted by aluminum, a low density and low atomic number material (Fig. 3).

The aluminum top wall of the light guide is $10 \mu \mathrm{m}$ thick. The X-ray intensity absorbed at $20 \mathrm{keV}$ is only $1 \%$, as it is shown in the graph of Fig. 3. The scintillator thickness is $800 \mu \mathrm{m}$, absorbing almost all of the $20 \mathrm{keV} \mathrm{X}$-ray photons, as it is shown in Fig. 4.

Without the aluminum walls, the spatial resolution of the $\mathrm{X}$-ray detectors is identical to the scintillator thickness [1], so one can expect a blur diameter of approximately $800 \mu \mathrm{m}$. By using the aluminum walls, each pixel spans a $540 \mu \mathrm{m} \times$ $540 \mu \mathrm{m}$ area $(400 \mu \mathrm{m}$ covered with scintillator plus $140 \mu \mathrm{m}$ for the aluminum walls). Therefore, the lateral spread of light is confined within approximately $58 \%$ of the blur area without the aluminum walls.

\section{Fabrication process}

The photodiodes are fabricated using a standard CMOS n-well $1.6 \mu \mathrm{m}$ process. This technology allows the accomplishment of four different types of photodiodes: three vertical structures and a lateral one [6]. Among the vertical diode structures, the junction $n+/$ substrate has the highest 


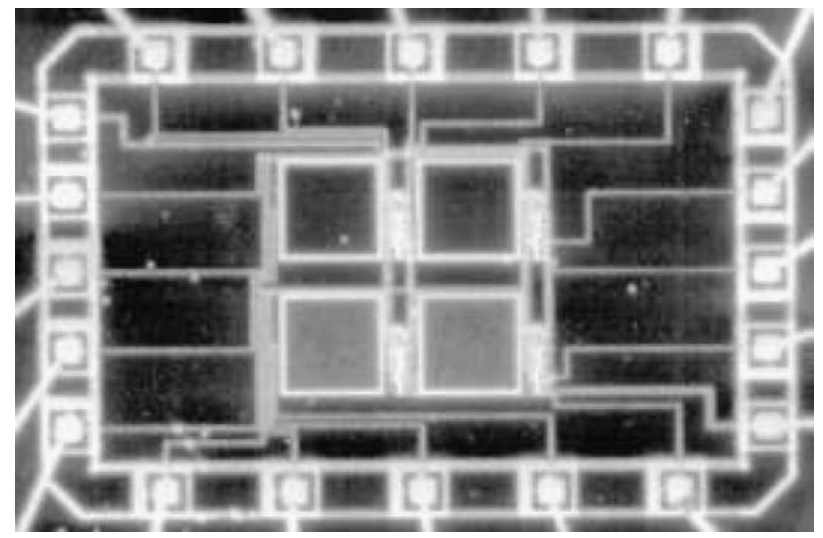

Fig. 5. Picture of a $2 \times 2$ photodiode array.

quantum efficiency and it has a better response for green wavelengths. The diode $\mathrm{p}+/ \mathrm{n}$-well has a better response for blue wavelengths relatively to the $n$-well/substrate diode, because its junction is closer to the surface and it can absorb more photons of short wavelength, where silicon has an high absorption coefficient. For a similar reason, the diode structure n-well/substrate is good for longer wavelengths, i.e., the red region. The lateral diode structure has a good response for the blue wavelengths, because the electron-hole pairs generated by these wavelengths contributes in a significative way to the output current. This is due to the fact that in this photodetectors, the junction goes to the surface, and the blue light is absorbed near it.

The photodiode structure chosen for this work was the $\mathrm{n}+/$ substrate due to its highest quantum efficiency and to its spectral response in the green region of the spectrum. As it will be shown, the light yielded by the scintillating crystal used in this work is green. For some specific applications, other type of photodiode can be combined with scintillator materials which produce light of different colors. A picture of the $2 \times 2$ photodiode array is shown in Fig. 5 .

In order to fabricate the light guides, $400 \mu \mathrm{m}$ diameter cavities were drilled in a $800 \mu \mathrm{m}$ thick aluminum sheet.

The scintillating crystal chosen for this work was the cesium iodide doped with thallium (CsI:Tl) due to its high light yield, reasonable decay time and emission wavelength in the green region. Table 1 shows some of the relevant properties of CsI:Tl. The scintillator was placed inside the aluminum cavities by a clamping pressure of about $10 \mathrm{MPa}$. This pressure is applied using the machine of Fig. 6, whose working principle is the following:

- The CsI:Tl is placed above the aluminum cavities. On the CsI:Tl lays a system constituted by two plates joined by springs. When the step motor rotates, the screw pushes the upper plate and compresses the springs, which push the lower plate. From the compression distance of the springs (measured with the scales), its elastic constant and the surface area of the CsI:Tl, it is possible to calculate the exerted pressure. In order to ensure that the cavities are
Table 1

Properties of CsI:Tl

\begin{tabular}{lc}
\hline Crystal class and space group & Cubic Pm3m (22 1) \\
Unit cell lattice parameters $(\AA)$ & 4.566 \\
Formulas per unit cell, $Z$ & 1 \\
Molecular weight $(\mathrm{amu})$ & 259.81 \\
Density (g/cm ${ }^{3}$ ) & 4.53 \\
Effective atomic number & 54 \\
Melting point $(\mathrm{K})$ & 898 \\
Cold water solubility (g/100 g) & 44.0 \\
Elastic moduli (GPa) & 18 \\
Shear moduli $(\mathrm{GPa})$ & 7.3 \\
Bulk moduli $(\mathrm{GPa})$ & 12.6 \\
Poisson's ratio & 0.26 \\
Flexure strength (MPa) & 5.6 \\
Light yield (phot/MeV) & 65900 \\
Emission wavelength (nm) & 560 \\
Decay time (ns) & $10^{3}$
\end{tabular}

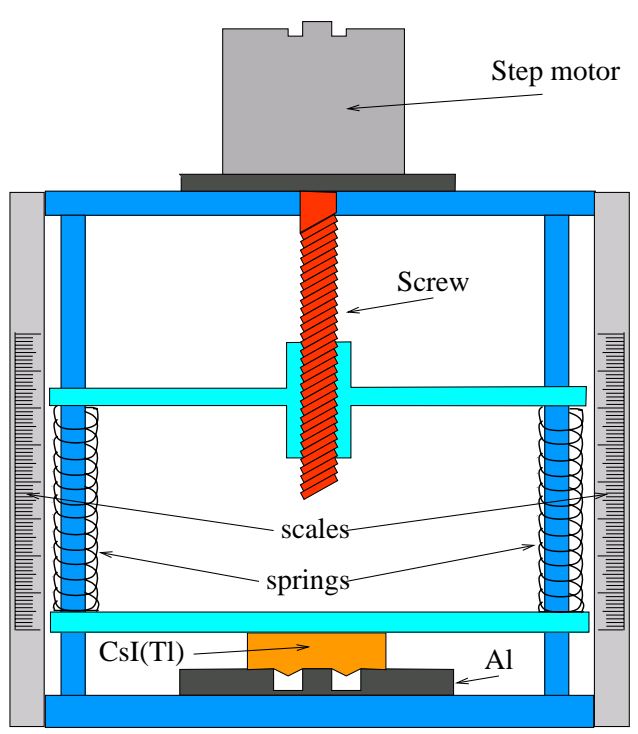

Fig. 6. Scintillator clamping system

well filled, the system of Fig. 6 is placed inside a vacuum chamber. The air inside the cavities is first removed and the CsI:Tl is then placed.

Fig. 7 shows the CsI:Tl crystals inside the aluminum cavities. A film of aluminum $(10 \mu \mathrm{m})$ is then placed on the top of the aluminum cavities filled with CsI:Tl. As a final step, the light guides setup is placed on the top of the photodiodes (Fig. 8).

\section{Experimental setup and results}

\subsection{Spectral response of the photodiodes}

The spectral response of the photodiodes was measured using an Oriel spectral analyzer system motorized monochromator UV-Vis. The output current was measured 


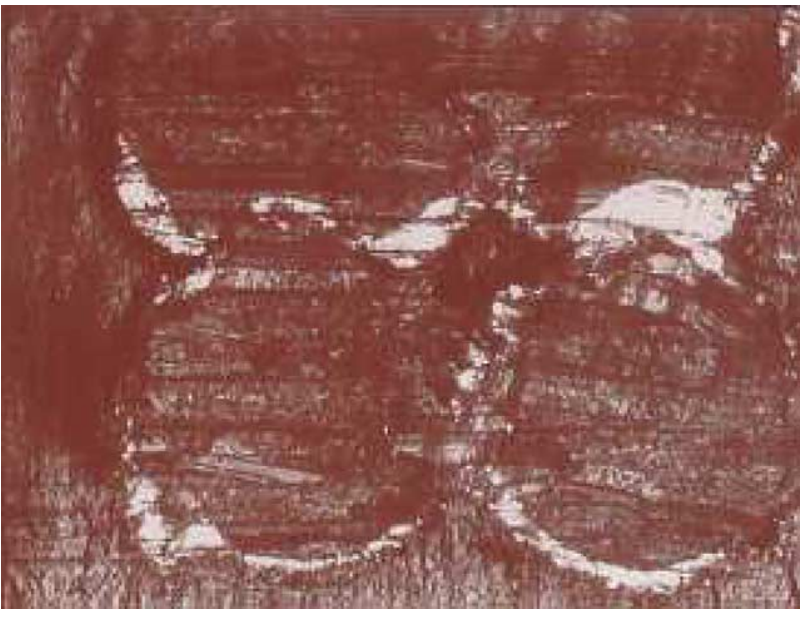

Fig. 7. CsI:Tl inside the aluminum holes: $2 \times 2$ array.

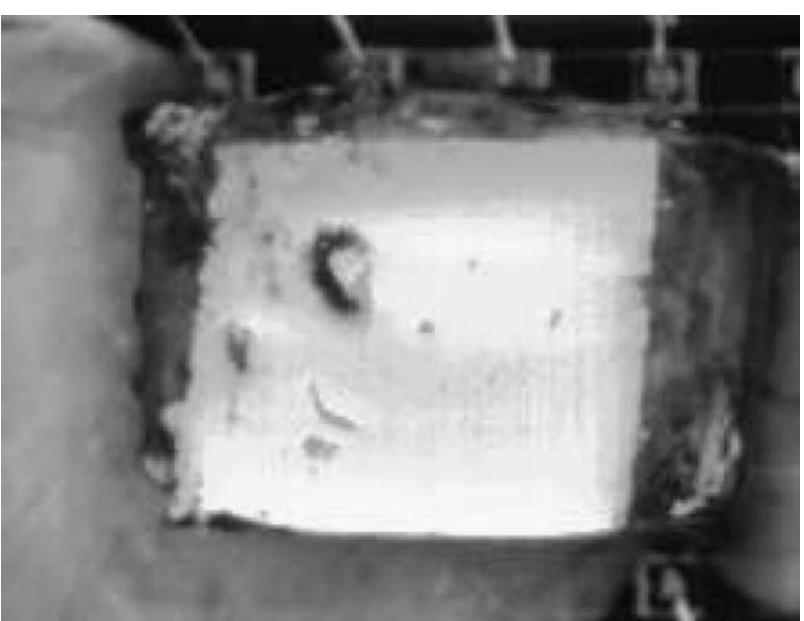

Fig. 8. Picture of the final prototype.

using a Keithley 487 picoamperimeter/voltage source. To perform this test, no bias was applied to the photodiode. Fig. 9 shows the measured current for each wavelength. The photodiode peek response is in the green region between 480 and $580 \mathrm{~nm}$.

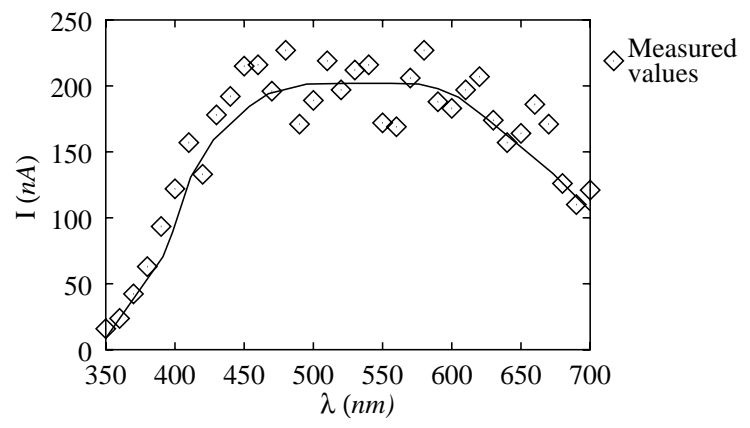

Fig. 9. Spectral response of the photodiodes.

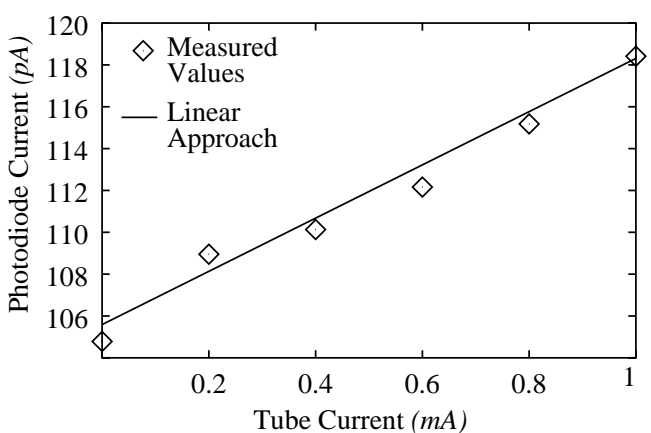

Fig. 10. Current detected for the photodiodes with an X-ray tube input of $35 \mathrm{kV}$ and different currents.

\subsection{X-ray detection}

The experimental test was performed using an X-ray tube (Leybold) with molybdenum anode.

The X-rays produced in this tube are composed by [7]:

- Braking radiation. According to the classic electrodynamics, an electron whose speed suffers a sudden reduction, emits radiation, preponderantly perpendicular to the direction of the acceleration.

- Characteristic radiation. When the energy of the electrons is high enough, the spectrum of the braking radiation is overlapped by a relatively simple structure of lines. This lines are characteristic of the anode material and are due to electronic transitions from the $\mathrm{L}$ and $\mathrm{M}$ shells to the $\mathrm{K}$ shell.

The molybdenum anode has a characteristic short wave radiation of $K_{\alpha}=17.4 \mathrm{keV}$ and $K_{\beta}=19.6 \mathrm{keV}$. The tube was powered with a voltage of $35 \mathrm{kV}$, and a current ranging from 0 to $1 \mathrm{~mA}$. Fig. 10 shows the measured values for one photodiode. The other three photodiodes show similar results. It is possible to observe that the measured results are close to the linear approach also drawn in Fig. 10.

In the second test the tube input power was constant $(35 \mathrm{kV}, 1 \mathrm{~mA})$. Several aluminum sheets with different thickness were placed between the $\mathrm{X}$-rays source and the sensor. The results are shown in Fig. 11. The measured values are represented by squares. The filled line was drawn according

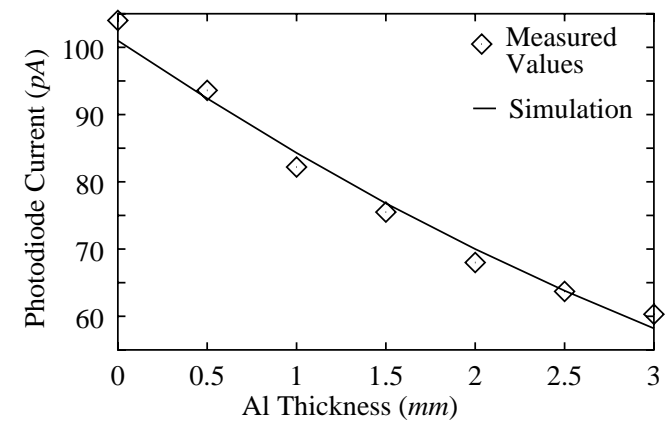

Fig. 11. Current detected for the photodiodes with an X-ray tube input of $35 \mathrm{kV}, 1 \mathrm{~mA}$ and different aluminum thickness. 
to an equation similar to the Beer-Lambert optical law:

$I=I_{0} \mathrm{e}^{(-\mu / \rho) \rho x}$,

where $I_{0}$ is the X-ray input intensity, $I$ the X-ray intensity at the distance $x$ from the surface, $\mu / \rho$ the mass-absorption coefficient and $\rho$ the density of the material. Once again, the experimental results follow closely the calculations.

\section{Conclusions}

This approach, scintillating light guides plus CMOS photodiodes, reveals to be suitable for X-ray imaging applications. The scintillating light guides allow a high scintillator thickness with low cross-talk and good spatial resolution. CMOS technology allows to implement X-ray detectors which reveal several advantages relative to the CCD technology. The X-ray source used to perform the presented tests is very weak when compared with the X-ray sources used on most medical and industrial applications nowadays. The obtained results allows to conclude that radiation dose can be reduced. As a future work photodiodes and readout electronics will be integrated in the same device.

\section{Acknowledgements}

This work was supported by The Foundation of Science and Technology, Portugal, FCT-CTM/POCTI/33751/1999; Grant-BD SFRH/BD/1296/2000.

\section{References}

[1] P. Kleymann, J. Linnros, et al., An X-ray imaging pixel detector based on a scintillating guides screen, IEEE Trans. Nucl. Sci. 47 (4) (2000) $1483-1486$.

[2] V.V. Nagarkar, S.V. Tipnis, et al., High speed X-ray imaging camera using a structured $\mathrm{CsI}(\mathrm{Tl})$ scintillator, IEEE Trans. Nucl. Sci. 46 (1999) 232-236.

[3] V.V. Nagarkar, T.K. Gupta, et al., Structured CsI(Tl) scintillators for X-ray imaging applications, IEEE Trans. Nucl. Sci. 45 (1998) 492496.

[4] S.T. Smith, D.R. Bednarek, et al., Evaluation of a CMOS image detector for low cost and power medical X-ray imaging applications, Proc. SPIE 3659 (1999) 952-961.

[5] J.G. Rocha, J.H. Correia, A high-performance scintillator-silicon-well $\mathrm{X}$-ray microdetector based on DRIE techniques, Sens. Actuators A 92 (2001) 203-207.
[6] A. Moini, Vision Chips, Kluwer Academic Publishers, Boston, 2000.

[7] C. Gerthsen, Kneser, H. Vogel, Physik. Ein Lehrbuch zum Gebrauch neben Vorlesungen. 17. Auflage, Springer-Verlag, Berlin, 1993.

\section{Biographies}

J.G. Rocha graduated in industrial electronics engineering in 1995 and obtained his MSc degree in 1999 both titles at the University of Minho, Portugal. Since 1996 he has been a Lecturer in the Department of Industrial Electronics, University of Minho, Portugal, and he is involved in the research of radiation sensors.

N.F. Ramos graduated in industrial electronics engineering in 2001 at the University of Minho. Since then he works in the Department of Industrial Electronics, University of Minho, Portugal, and he is involved in research of radiation sensors.

S. Lanceros-Mendez graduated in physics at the University of the Basque Country, Leioa, Spain, in 1991. He obtained his PhD degree in 1996 at the Institute of Physics of the Julius-Maximilians-Universität Würzburg, Germany. He was Research Scholar at Montana State University, Bozeman, MT, from 1996 to 1998. Since September 1998 he has been Assistant Professor at the Physics Department of the University of Minho, Portugal, where he is involved in experimental investigations in the area of Condensed Matter Physics and its applications. He was a Visitor at the A.F. Ioffe Physico-Technical Institute, St. Petersburg, Russia, in 1995 and at the University of Porto, Portugal, in 1996.

R.F. Wolffenbuttel received his $\mathrm{MSc}$ and $\mathrm{PhD}$ degrees from the Delft University of Technology, Delft, The Netherlands, in 1984 and 1988, respectively. Between 1986 and 1993, he was an Assistant Professor, and since 1993, an Associate Professor, at the Laboratory of Electronic Instrumentation of the Delft University of Technology, where he is involved in instrumentation and measurement in general and on-chip functional integration of microelectronic circuits and silicon sensor, fabrication compatibility issues, and micromachining in silicon and microsystems, in particular. He was a Visitor at the University of Michigan, Ann Arbor, in 1992, 1999, and 2001, at Tohoku University, Sendai, Japan, in 1995, and at EPFL, Lausanne, Switzerland, in 1997. Dr. Wolffenbuttel was the recipient of a 1997 NWO pioneer award. He served as General Chairman of the Dutch National Sensor Conference in 1996 and Eurosensors in 1999.

J.H. Correia graduated in Physical Engineering at the University of Coimbra, Portugal, in 1990. He obtained his PAPCC (equivalent to MSc degree) in 1994 at the University of Minho, Portugal, and his PhD degree in 1999 at the Delft University of Technology, The Netherlands. His thesis work dealt with the development of optical microsystems in silicon for the visible part of the spectrum. Since May 1999 he has been an Assistant Professor at the Department of Industrial Electronics of the University of Minho and he is involved in instrumentation and measurement in general and on-chip functional integration of microelectronic circuits and silicon sensors. 